\title{
PRODUÇÃO DE PAINEL COMPENSADO ESTRUTURAL DE Eucalyptus grandis E Eucalyptus dunnii
}

\author{
Setsuo Iwakiri*, Sidon Keinert Jr.**, José Guilherme Prata***, Silviana Rosso*** \\ *Eng. Florestal, Dr., Depto. de Engenharia e Tecnologia Florestal, UFPR - setsuo@ufpr.br \\ **Eng. Florestal, Ph.D., Depto. de Engenharia e Tecnologia Florestal, UFPR - sidonk@terra.com.br \\ ***Eng. Florestal, Doutorando em Engenharia Florestal, UFPR - jgprata@gmail.com - silviana.rosso@gmail.com \\ Recebido para publicação: 22/09/2006 - Aceito para publicação: 04/06/2007
}

\begin{abstract}
Resumo
Este trabalho foi desenvolvido com o objetivo de avaliar o potencial de utilização de lâminas de Eucalyptus grandis e Eucalyptus dunnii para produção de painéis compensados estruturais. Os painéis foram produzidos com diferentes formulações de adesivo à base de resina fenol-formaldeído. Os resultados indicaram que as diferentes formulações do adesivo não afetam de forma clara a resistência da linha de cola. Entre as espécies, os painéis de E. grandis apresentaram maiores valores de resistência da linha de cola em comparação aos painéis de E. dunnii. Os valores médios de MOR das duas espécies foram estatisticamente iguais. Os resultados das propriedades mecânicas avaliadas apontam que o $E$. grandis e E. dunnii são espécies com grande potencial para produção de painéis compensados estruturais. Palavras-chave: Compensado; eucalipto; lâminas de madeira.
\end{abstract}

\begin{abstract}
Production of structural plywood from Eucalyptus grandis and Eucalyptus dunnii. This research was developed with the objective to evaluate the utilization of $E$. grandis and $E$. dunnii veneer for production of structural plywood. The experimental panels were manufactured using different formulations of phenol-formaldehyde adhesive. The results showed that the different adhesive formulation did not affect the glue line strength. Plywood manufactured from $E$. grandis presented greater glue line strength values than those from $E$. dunnii. The MOR values for of $E$. grandis and $E$. dunnii were statistically equal. The results indicate that both species have potential for production of structural plywood.

Keywords: Plywood; eucalipto; veneer.
\end{abstract}

\section{INTRODUÇÃO}

A partir da década de 80 , as indústrias de base florestal no Brasil passaram por uma grande transformação em termos de matéria-prima, produtos e processos produtivos. A base da matéria-prima madeira, que era quase que totalmente oriunda de florestas nativas, passou a ser substituída pelas florestas plantadas de rápido crescimento, especialmente a de pinus e eucalipto. As espécies do gênero Pinus (taeda e elliottii) foram as primeiras a serem utilizadas para a substituição de madeiras nativas na fabricação de produtos sólidos e reconstituídos, em face das suas características tecnológicas e disponibilidade (ABIMCI, 2004).

Atualmente, a madeira de pinus é amplamente utilizada na fabricação de madeiras serradas e beneficiadas, laminados e compensados, aglomerados, MDF e OSB. Entretanto, as estatísticas recentes têm demonstrado que a relação oferta/demanda de madeira de pinus está atingindo a exaustão e deverá em poucos anos, faltar matéria-prima em condições adequadas para processamento industrial. Essa situação está sendo chamada de "apagão florestal".

Dentro desse cenário, as empresas florestais têm procurado, nas espécies do gênero eucalipto, uma alternativa para fornecimento de matéria-prima. Entre as espécies de eucalipto, cabe destacar a madeira de Eucalyptus grandis, em função das altas taxas de produtividade das florestas plantadas, da boa adaptação em grande parte do território brasileiro e da relativa disponibilidade de florestas para pronto uso (SILVA, 2002). É uma espécie que já vem sendo utilizada, ainda que em pequena escala, para produção de madeira serrada, laminas e compensados. 
A outra espécie promissora que apresenta grandes vantagens quanto aos aspectos silviculturais é Eucalyptus dunnii. Trata-se de uma espécie de rápido crescimento e que se adaptou satisfatoriamente nas regiões de ocorrência de geadas e inverno rigoroso como nos Estados da região sul do Brasil. A sua madeira já é utilizada pelas indústrias de Santa Catarina, principalmente na forma de serrados. Uma das limitações para uso da madeira de Eucalyptus dunnii, assim como da maioria das espécies de eucalipto, são as altas tensões internas de crescimento. O seu uso na forma de madeira sólida é muito prejudicado em função da alta instabilidade dimensional e baixo rendimento das toras (ROCHA, 2000). Para minimizar os efeitos da instabilidade dimensional das peças, uma das alternativas seria a fabricação de produtos reconstituídos de madeira através do processo de colagem de lâminas e partículas para obtenção de painéis compensados e particulados.

O compensado estrutural é classificado como de uso exterior (EX) e trata-se de um painel multilaminado, colado com resinas à prova d'água (normalmente fenol-formaldeído), destinadas a uso em condições cíclicas de alta e baixa umidade relativa, eventualmente em ação direta com a água. A sua aplicação se destina principalmente ao setor de construção civil e para embalagens (BALDWIN, 1995).

$\mathrm{O}$ uso da resina fenol-formaldeído confere ao compensado alta resistência à ação da umidade e água, em função da sua composição química. O processo de prensagem à alta temperatura requer alguns cuidados especiais, como controle do teor de umidade das lâminas do miolo, que deve estar abaixo de 8\% para evitar a formação de "bolhas" e delaminações no painel, em função da alta pressão interna de vapor gerada entre as linhas de colagem das lâminas (MARRA, 1992).

Quanto às espécies, as madeiras de Eucalyptus grandis e Eucalyptus dunnii são consideradas de média densidade, tornando-se uma vantagem técnica significativa na fabricação de painéis compensados para fins estruturais, que requerem maior resistência mecânica. Marra (1992) e Tsoumis (1991) relatam que as madeiras com maior densidade apresentam menor porosidade, o que irá influenciar diretamente na redução da penetrabilidade do adesivo durante a formação da ligação adesiva. Segundo autores, a formulação do adesivo deve ser ajustada para adequação das condições de colagem em função da densidade da madeira e finalidade de uso dos painéis.

Este trabalho teve como objetivo avaliar a qualidade de painéis compensados estruturais, produzidos com madeiras de Eucalyptus grandis e Eucalyptus dunnii em diferentes formulações do adesivo à base de fenol-formaldeído.

\section{MATERIAIS E MÉTODOS}

Foram utilizadas nesta pesquisa lâminas de madeiras de Eucalyptus grandis e Eucalyptus dunnii, obtidas de árvores com 15 e 18 anos, de plantios localizados respectivamente nas regiões de Telêmaco Borba (PR) e Três Barras (SC). Para a colagem de lâminas, foi utilizada a resina fenol-formaldeído, com teor de sólidos de $49 \%$ e viscosidade Brookfield de $500 \mathrm{cP}$.

As lâminas com 2,0 mm de espessura e dimensões laterais de 50 x $50 \mathrm{~cm}$ foram secadas em estufa de laboratório até o teor de umidade médio de $6 \%$. Os painéis compensados foram produzidos com cinco lâminas, sendo dois painéis por tratamento, conforme demonstrado no delineamento experimental da tabela 1.

Tabela 1. Delineamento experimental.

Table 1. Experimental design.

\begin{tabular}{lll}
\hline Tratamento & Espécie & Formulação do adesivo \\
\hline $\mathrm{T} 1$ & E. grandis & $\mathrm{A} \quad \mathrm{R}=100 ; \mathrm{E}=5 ; \mathrm{C}=5 ; \mathrm{A}=5 \ldots \ldots . \mathrm{TS}=42,6 \%$ \\
$\mathrm{~T} 2$ & E. grandis & $\mathrm{B} \quad \mathrm{R}=100 ; \mathrm{E}=10 ; \mathrm{A}=10 \ldots \ldots \ldots \ldots \mathrm{TS}=40,8 \%$ \\
$\mathrm{~T} 3$ & E. grandis & $\mathrm{C} \quad \mathrm{R}=100 ; \mathrm{E}=15 ; \mathrm{A}=10 \ldots \ldots \ldots \ldots \mathrm{TS}=37,7 \%$ \\
$\mathrm{~T} 4$ & $\mathrm{~A} \quad \mathrm{R}=100 ; \mathrm{E}=5 ; \mathrm{C}=5 ; \mathrm{A}=5 \ldots \ldots . \mathrm{TS}=42,6 \%$ \\
$\mathrm{~T} 5$ & E. dunnii & $\mathrm{B} \quad \mathrm{R}=100 ; \mathrm{E}=10 ; \mathrm{A}=10 \ldots \ldots \ldots . \mathrm{TS}=40,8 \%$ \\
$\mathrm{~T} 6$ & E. dunnii & $\mathrm{C} \quad \mathrm{R}=100 ; \mathrm{E}=15 ; \mathrm{A}=10 \ldots \ldots \ldots . \mathrm{TS}=37,7 \%$ \\
\hline
\end{tabular}

R: resina fenol-formaldeído; E: extensor - farinha de trigo; C: farinha de casca de coco; A: água; TS: teor de sólidos da batida de cola.

A batida de cola foi preparada com diferentes partes de resina fenol-formaldeído, extensor (trigo), farinha de casca de coco e água, conforme as formulações A, B e C apresentadas na Tabela 1. 
O adesivo foi aplicado manualmente sobre a superfície das lâminas com gramatura de $160 \mathrm{~g} / \mathrm{m}^{2}$ (linha simples), e os painéis foram prensados à temperatura de $150{ }^{\circ} \mathrm{C}$, pressão específica de $15 \mathrm{kgf} / \mathrm{cm}^{2} \mathrm{e}$ tempo de permanência na prensa de 10 minutos.

Após a prensagem, os painéis foram pré-esquadrejados e acondicionados na câmara climática à temperatura de $20 \pm 1{ }^{\circ} \mathrm{C}$ e umidade relativa de $65 \pm 3 \%$, até a sua estabilização. Para a realização dos ensaios, foram retirados, de cada painel, quatro corpos de prova, para ensaios de flexão estática paralela, e 10 corpos de prova para ensaios de resistência da linha de cola às tensões de cisalhamento, sendo cinco para teste seco e cinco para teste de fervura. Os ensaios foram realizados de acordo com a Norma Européia EN 314 - 1993 para resistência da linha de cola e EN 310 - 1993 para flexão estática.

Os resultados foram analisados estatisticamente através de ANOVA e teste de Tukey ao nível de probabilidade de $95 \%$.

\section{RESULTADOS E DISCUSSÃO}

\section{Densidade das lâminas}

Os resultados referentes às densidades das lâminas de E. grandis e E. dunnii estão apresentados na tabela 2.

Tabela 2. Densidade das lâminas de E. grandis e E. dunnii.

Table 2. Density of E. grandis and E. dunnii veneers.

\begin{tabular}{lcc}
\hline \multirow{2}{*}{ Densidade $\left(\mathbf{g} / \mathbf{c m}^{\mathbf{3}}\right)$} & \multicolumn{2}{c}{ Espécie } \\
\cline { 2 - 3 } & $\boldsymbol{E .}$ grandis & $\boldsymbol{E . ~ d u n n i i}$ \\
\hline Valor médio & 0,487 & 0,593 \\
Valor mínimo & 0,383 & 0,456 \\
Valor máximo & 0,554 & 0,738 \\
\hline
\end{tabular}

Os valores médio e máximo da densidade das lâminas de E. dunnii foram bem superiores em comparação às lâminas de E. grandis, confirmando os resultados apresentados por vários pesquisadores. Rocha (2000) encontrou, para madeiras de E. grandis e E. dunnii, valores médios de densidade aparente em $12 \%$ de umidade de $0,55 \mathrm{~g} / \mathrm{cm}^{3}$ e $0,74 \mathrm{~g} / \mathrm{cm}^{3}$, respectivamente. A maior densidade das lâminas de $E$. dunnii poderá influenciar na formação da ligação adesiva entre as lâminas em função da penetração do adesivo na madeira ser dificultada. Segundo Marra (1992), a maior densidade da madeira pode afetar também em termos de geração de maior pressão interna de vapor durante o processo de prensagem à alta temperatura, contribuindo para a redução da taxa de polimerização da resina. Por outro lado, a maior densidade da madeira reflete diretamente no aumento da resistência do painel à flexão estática.

\section{Resistência da linha de cola às tensões de cisalhamento}

Os resultados da resistência da linha de cola apresentados na tabela 3 indicam que, tanto para $E$. grandis quanto para $E$. dunnii, os valores médios referentes ao teste seco obtidos para os painéis produzidos com diferentes teores de sólidos do adesivo (Formulação A, B, C) não diferiram estatisticamente no nível de probabilidade de $95 \%$. Para o teste de fervura, os painéis de E. grandis produzidos com formulação A, com maior teor de sólidos, apresentou valor médio de resistência da linha de cola estatisticamente inferior em relação aos painéis produzidos com formulação C. Para E. dunnii, não foram constatadas diferenças estatisticamente significativas entre as médias dos tratamentos com diferentes formulações.

De uma maneira geral, os resultados indicam que não houve influência significativa de diferentes teores de sólidos da batida de cola (formulações A, B, C) na resistência da linha de cola dos painéis produzidos com lâminas de E. grandis e E. dunnii. Essa constatação é muito importante sob o ponto de vista econômico, tendo em vista que a redução da proporção de resina fenol-formaldeído na composição da batida de cola não reduziu os valores de resistência da linha de cola dos painéis.

Os conceitos apresentados por Marra (1992) podem explicar as tendências observadas para aumento da resistência da linha de cola com a redução do teor de sólidos da batida de cola, como relacionadas à redução na sua viscosidade, resultando em melhor "ancoragem" entre a linha de cola e a superfície de colagem das lâminas. Esse mesmo conceito da "ancoragem" pode ser aplicado para os painéis de E. dunnii, produzidos com lâminas mais densas, que apresentaram valores inferiores de 
resistência da linha de cola em comparação aos painéis de E. grandis. Nesse caso, a madeira mais densa apresenta menor porosidade, prejudicando as ações de mobilidade do adesivo e o processo de "ancoragem" entre a linha de cola e as lâminas adjacentes.

Tabela 3. Resistência da linha de cola - teste seco e fervura.

Table 3. Glue line strength - dry and boil tests.

\begin{tabular}{|c|c|c|c|c|}
\hline \multirow{2}{*}{$\begin{array}{l}\text { Tratamento } \\
\text { Espécie/Formulação }\end{array}$} & \multicolumn{2}{|c|}{ Teste seco } & \multicolumn{2}{|c|}{ Teste fervura } \\
\hline & Média $\left(\mathrm{kgf} / \mathrm{cm}^{2}\right)$ & CV (\%) & Média $\left(\mathrm{kgf} / \mathrm{cm}^{2}\right)$ & CV (\%) \\
\hline T1-grandis/A & $29,02 \mathrm{~cd}$ & 8,83 & $14,08 \mathrm{ab}$ & 12,31 \\
\hline T2-grandis/B & $26,95 \mathrm{bcd}$ & 16,28 & $15,76 \mathrm{bc}$ & 28,31 \\
\hline $\mathrm{T} 3$-grandis/C & $31,17 \quad d$ & 15,29 & $19,38 \quad \mathrm{c}$ & 19,49 \\
\hline T4-dunnii/A & $21,85 \mathrm{a}$ & 8,92 & $10,87 \mathrm{a}$ & 23,90 \\
\hline T5-dunnii/B & $24,34 \mathrm{ab}$ & 10,88 & $12,01 \mathrm{ab}$ & 19,60 \\
\hline T6-dunnii/C & $25,19 \mathrm{abc}$ & 13,79 & $14,88 \mathrm{ab}$ & 25,77 \\
\hline
\end{tabular}

Médias seguidas de mesma letra são estatisticamente iguais no nível de probabilidade de $95 \%$.

Todos os valores médios de resistência da linha de cola às tensões de cisalhamento obtidos para as duas espécies atendem aos requisitos mínimos exigidos pela norma EN 314 (1993), de 1,0 MPa ou $10,197 \mathrm{kgf} / \mathrm{cm}^{2}$.

\section{Flexão estática}

Os valores médios de MOE em flexão estática, apresentados na Tabela 4, demonstram que, tanto para $E$. grandis quanto para $E$. dunnii, apenas o tratamento T2, referente aos painéis de E. grandis produzidos com formulação $\mathrm{B}$, apresentou valor médio estatisticamente inferior em comparação aos painéis produzidos com formulação $\mathrm{A}$ e $\mathrm{C}$, respectivamente, com maior e menor teor de sólidos.

Com relação ao MOR, para as duas espécies, não foram constatadas diferenças estatísticas entre as médias obtidas para painéis produzidos com diferentes formulações do adesivo. Esse resultado é importante sob o ponto de vista econômico, tendo em vista que dentro da faixa de teor de sólidos definida neste estudo, o aumento na proporção de resina fenol-formaldeído na batida de cola não resultou em aumento significativo da resistência dos painéis nos ensaios de flexão estática.

Tabela 4. Módulo de elasticidade (MOE) e módulo de ruptura (MOR) em flexão estática.

Table 4. Modulus of elasticity (MOE) and modulus of rupture (MOR) in static bending.

\begin{tabular}{lllccc}
\hline Tratamento & \multicolumn{3}{c}{ MOE $\mathbf{( k g f / \mathbf { c m } ^ { 2 } )}$} & \multicolumn{2}{c}{ MOR (kgf/cm $\mathbf{~})$} \\
\cline { 2 - 6 } Espécie/Formulação & Média & $\mathbf{C V ~ ( \% )}$ & Média & CV (\%) \\
\hline T1-grandis/A & 123.100 & $\mathrm{~b}$ & 5,52 & $762 \mathrm{a}$ & 15,08 \\
T2-grandis/B & $110.580 \mathrm{a}$ & 7,08 & $837 \mathrm{a}$ & 9,76 \\
T3-grandis/C & 128.542 & $\mathrm{~b}$ & 4,58 & $804 \mathrm{a}$ & 10,86 \\
T4-dunnii/A & 133.863 & $\mathrm{~b}$ & 8,55 & $753 \mathrm{a}$ & 13,67 \\
T5-dunnii/B & 126.996 & $\mathrm{~b}$ & 3,53 & $775 \mathrm{a}$ & 5,73 \\
T6-dunnii/C & 126.955 & $\mathrm{~b}$ & 7,77 & $738 \mathrm{a}$ & 9,52 \\
\hline
\end{tabular}

Médias seguidas de mesma letra são estatisticamente iguais no nível de probabilidade de $95 \%$.

Com relação às espécies, os painéis de $E$. dunnii produzidos com a formulação $\mathrm{B}$ apresentaram valor médio de MOE estatisticamente superior em comparação aos painéis de $E$. grandis produzidos com a mesma formulação do adesivo. Para esses tratamentos, a maior densidade das lâminas de $E$. dunnii teve influência significativa na rigidez dos painéis compensados. Para os painéis produzidos com formulações A e C, não foram constatadas diferenças estatisticamente significativas e tãopouco foram verificadas tendências definidas de aumento ou redução entre os valores médios de MOE obtidos para as duas espécies.

Para o MOR, os valores médios obtidos para as duas espécies não diferiram estatisticamente entre si para as três formulações avaliadas. Entretanto, os valores médios obtidos para as duas espécies indicam uma tendência de redução dessa propriedade para os painéis produzidos com lâminas de $E$. dunnii, embora a sua densidade seja maior em relação às lâminas de E. grandis. Essa diferença pode ser atribuída, em parte, aos menores valores médios de resistência da linha de cola dos painéis de E. dunnii, 
resultante da menor mobilidade do adesivo no processo de formação da ligação adesiva entre as lâminas, em função da maior densidade e menor porosidade da sua madeira.

Os valores médios de MOE e MOR dos painéis de E. grandis e E. dunnii obtidos nesta pesquisa foram expressivamente superiores aos valores médios de $85.477 \mathrm{kgf} / \mathrm{cm}^{2}$ para MOE e $498 \mathrm{kgf} / \mathrm{cm}^{2}$ para MOR, referenciados no catálogo técnico da ABIMCI (2002), para painéis compensados estruturais de $P$. taeda de padrão exportação. Em comparação com os resultados encontrados por Pedrosa (2002) para painéis compensados de E. dunnii, cujos valores de MOE e MOR foram respectivamente de $132.814 \mathrm{kgf} / \mathrm{cm}^{2}$ e 669 $\mathrm{kgf} / \mathrm{cm}^{2}$, os resultados desta pesquisa podem ser considerados satisfatórios, principalmente em relação ao MOR.

\section{CONCLUSÕES} apresentadas:

Com base nos resultados obtidos nesta pesquisa, as seguintes conclusões podem ser

- Os painéis de E. dunnii apresentaram menor resistência da linha de cola às tensões de cisalhamento em comparação aos painéis de E. grandis.

- Os resultados dos ensaios de resistência da linha de cola às tensões de cisalhamento obtidos para as duas espécies, atendem aos requisitos mínimos exigidos pela norma EN 314 (1993) de 1,0 MPa ou $10,197 \mathrm{kgf} / \mathrm{cm}^{2}$.

- As diferentes formulações do adesivo com variações no teor de sólidos da batida de cola, não afetaram de forma significativa as propriedades de resistência mecânica dos painéis.

- Os valores médios de MOR obtidos para as duas espécies foram estatisticamente iguais.

- Os valores médios de MOE e MOR em flexão estática obtidos para os painéis de E. grandis e E. dunnii foram expressivamente superiores aos valores médios referenciados para painéis comerciais de $P$. taeda destinados para uso estrutural, sendo um indicativo do potencial dessas espécies para a produção de painéis compensados estruturais.

\section{REFERÊNCIAS}

ASSOCIAÇÃO BRASILEIRA DA INDÚSTRIA DA MADEIRA PROCESSADA MECANICAMENTE.

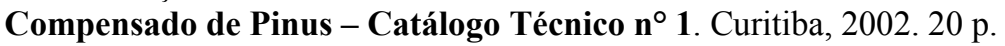

ASSOCIAÇÃO BRASILEIRA DA INDÚSTRIA DA MADEIRA PROCESSADA MECANICAMENTE. Estudo Setorial 2004. Curitiba, 2005. 50 p.

BALDWIN, R. F. Plywood and veneer-based products: manufacturing practices. San Francisco: M. Freeman, 1995. 388 p.

\section{EUROPEAN COMMITTEE FOR STANDARDIZATION. Norma CEN. 1993.}

MARRA, A. A. Technology of wood bonding: principles in practice. New York: Van Nostrand Reinhold, 1992. 453 p.

PEDROSA, A. L. Desempenho estrutural de vigas "I" constituídas por PLP e compensado de Pinus taeda L. e Eucalyptus dunnii Maiden e OSB de Pinus spp. 104 f.. Dissertação (Mestrado em Ciências Florestais) - Setor de Ciências Agrárias, Universidade Federal do Paraná, Curitiba, 2003.

ROCHA, M. P. Eucalyptus grandis Hill ex Maiden e Eucalyptus dunnii Maiden como fontes de matéria-prima para serrarias. 185 f. Tese (Doutorado em Ciências Florestais) - Setor e Ciências Agrárias, Universidade Federal do Paraná, Curitiba, 2000.

SILVA, J. C. Caracterização da madeira de Eucalyptus grandis Hill ex. Maiden, de diferentes idades, visando a sua utilização na indústria moveleira. $160 \mathrm{f}$. Tese (Doutorado em Ciências Florestais) - Setor de Ciências Agrárias, Universidade Federal do Paraná, Curitiba, 2002.

TSOUMIS, G. Science and technology of wood: structure, properties and utilization. New York: Van Nostrand Reinhold, 1991. 494 p. 